\title{
Diferencias culturales entre países y acceso de la mujer a los consejos de administración
}

\section{Cultural differences across countries and access of woman to boards of directors}

\author{
Amalia Carrasco Gallego ${ }^{* *}$. Universidad de Sevilla \\ Joaquina Laffarga Briones. Universidad de Sevilla \\ Emiliano Ruiz Barbadillo. Universidad de Cádiz
}

RESUMEN Nuestro estudio pretende comparar el nivel de representación de las mujeres en los consejos de administración de cinco países, analizando especialmente si la cultura que prevalece en el país puede estar afectando a la capacidad de la mujer de acceder a los puestos, tradicionalmente reservados a los hombres, de los consejos de administración. Sirviéndonos de las dimensiones culturales de Hofstede (1980) y de GLOBE (House et al., 2004) nuestro estudio revela que la cultura del país, con independencia de la métrica que se utilice, tiene capacidad para explicar el diferente nivel de representación de las mujeres en los consejos de administración. En particular, tres de las cuatro dimensiones culturales se revelan como significativas: distancia en el poder, aversión a la incertidumbre y masculinidad.

PALABRAS CLAVE Cultura; Consejos de administración; Género; Comparación países; Gobierno corporativo.

\begin{abstract}
The purpose of this paper is to compare the levels of representation of women on boards of directors in five countries, and especially to determine if the culture of a country is a factor that influences the possibility of women achieving positions on boards of directors. We use the cultural dimensions of Hofstede (1980) and GLOBE (House et al., 2004), and the results show that the culture of a country can be a factor in explaining the different levels of representation of women at board of directors. In particular, three of the four cultural dimensions are seen to be significant: power distance, uncertainty avoidance and masculinity.
\end{abstract}

KEY WORDS Culture; Board of directors; Gender; Cross-country; Corporate governance.

\footnotetext{
* Agradecimiento: Este trabajo puede considerarse un resultado parcial del proyecto EC02010-21627 financiado por el Ministerio de Ciencia e Innovación y del proyecto de excelencia P08-SJ-03950 financiado por la Consejería de Innovación, Ciencia y Empresa de la Junta de Andalucía.

** Autora para correspondencia: Amalia Carrasco Gallego, Departamento de Contabilidad y Economía Financiera, Avenida Ramón y Cajal 11, 41018 Sevilla. Tel. 954557616, Fax 954557569. Correo-e: agallego@us.es
} 


\section{INTRODUCCIÓN}

En los últimos tiempos se ha acrecentado el interés por el tema de la igualdad de género en la sociedad adquiriendo especial relieve en relación a la estructura de poder de las organizaciones y en particular, en la representación de la mujer en los consejos de administración de las empresas. En este sentido, un volumen importante de estudios ponen de manifiesto que el acceso de la mujer a los puestos de la cúspide de las organizaciones es menor al de los hombres (Catalys, 2004, 2008; EPWN, 2004, 2008; Heidrick y Struggles, 2005, 2007).

Tal como señalan Terjesen et al. (2009) este fenómeno es global, si bien, la amplia mayoría de los estudios sobre diversidad de género y gobierno de las empresas se han enfocado a nivel micro, donde se analizan entre otras cuestiones las características de las mujeres miembros de los consejos de administración, como formación y experiencia profesional, y las características políticas y legales en la propiedad y el control, como variables que puedan explicar en un entorno particular el nivel de representación de la mujer en los consejos de administración.

La perspectiva de nuestro trabajo es diferente dado que pretendemos realizar una comparación en distintos países del nivel de representación de mujeres en los consejos de administración. A este respecto cabe resaltar que la evidencia empírica acumulada revela que el nivel de acceso de la mujer a estos consejos no resulta similar cuando se comparan distintos países. Incluso si nos situamos en países con niveles socioeconómicos muy similares, como podrían ser los países europeos, se encuentran grandes diferencias en este porcentaje de presencia de mujeres en los consejos de administración (Catalys 2008) ${ }^{(1)}$. Esta evidencia empírica genera interés por comprender cuáles son las razones que pueden explicar el diferente nivel de acceso de la mujer a los consejos de administración. La tesis que se sustenta en este trabajo es que el diferente nivel de representación de mujeres en los consejos de las empresas cuando se comparan países distintos viene explicado en gran parte por la cultura que prevalece en cada país, dado que ésta puede crear estereotipos de roles asociados a hombres y mujeres que pueden afectar al acceso de la mujer a los consejos de administración.

El concepto de cultura puede ser tratado en diferentes niveles, tal como a nivel individual, a nivel grupal, a nivel organizacional o a nivel nacional (Erez y Early, 1993). En este trabajo el concepto de cultura es tratado a un nivel nacional, partiendo de la premisa, tal como sugiere Hofstede (1991), que el país es una fuente importante del programa mental global de sus ciudadanos. En este sentido, la cultura de un país puede quedar definida como un programa mental colectivo que representa la programación mental compartida por los miembros de ese país, la cual se materializa en valores, creencias, asunciones, expectativas, actitudes y comportamientos comunes a los miembros de un país en concreto. De esta forma la perspectiva de género en un

(1) Por ejemplo para datos del 2008, destaca Noruega con $44,2 \%$ de mujeres representadas en el consejo de administración, Suecia con un 26,9\%, Finlandia con un $25 \%$, Dinamarca con un 18,1\%, Reino Unido con un 11,5\%, Bélgica con un $7 \%$, España con un $6,6 \%$ o Italia con un $2,1 \%$. 
país puede quedar afectada por la estructura mental a través de la cual los individuos piensan sobre su mundo social, generándose estereotipos de género o roles asociados a la mujer particulares que podrían influenciar la presencia de mujeres en los consejos de administración (Nelson y Levesque, 2007).

Al objeto de contrastar la tesis de que la cultura de un país puede explicar el distinto nivel de acceso de la mujer a los consejos de administración de las empresas, en este trabajo utilizamos el modelo cultural propuesto por Hofstede (1980, 1991, 2000). El modelo cultural de Hofstede parte de la existencia de una distancia cultural entre países, lo que explicaría que países distintos respondan de forma diversa ante un mismo fenómeno social. Esta distancia cultural puede ser medida empíricamente, para lo cual Hofstede desarrolló el concepto de dimensión cultural, que son los indicadores determinantes de la cultura de un país en comparación a la de otro país. Dichas dimensiones culturales fueron derivadas de forma empírica a través de las respuestas que los miembros de distintos países dieron a cuatro problemas básicos de toda sociedad como son el tratamiento que se le otorga a la desigualdad en la sociedad, la forma de relación entre individuo y grupo, el concepto de género y la actitud de la sociedad frente a la incertidumbre. La posterior agregación de las respuestas individuales en función del país de origen de los sujetos intervinientes en el estudio, posibilitó que Hofstede identificara cuatro dimensiones culturales que resultan determinantes de la cultura de un país, y son la base explicativa de la distancia cultural entre países. Estas dimensiones culturales fueron denominadas como: distancia al poder, que es el grado en el cual una sociedad acepta la distribución desigual del poder en organizaciones e instituciones; aversión a la incertidumbre, que representa como la sociedad se adapta al cambio y gestiona la incertidumbre; individualismo-colectivismo, que determina como son las relaciones entre individuos y grupos; y, por último, masculinidad que trata con las implicaciones sociales del género. Estas dimensiones culturales son indicadores de los valores agregados de una sociedad más que una medida de los valores individuales, permitiendo de esta forma realizar análisis comparativos entre países.

El estudio empírico es realizado sobre una muestra de empresas de cinco países distintos, en concreto Reino Unido, Bélgica, España, Dinamarca y Suecia. Tras obtener los datos sobre la representación de mujeres en los consejos de administración de estos países, los resultados que obtenemos ponen de relieve como la cultura que prevalece en estos países tiene capacidad para explicar el diferente nivel de representación de las mujeres en el consejo de administración, adquiriendo significado estadístico las dimensiones culturales distancia al poder, aversión a la incertidumbre y masculinidad. Nuestros resultados resultan igualmente robustos a especificaciones distintas de la muestra y del modelo utilizado.

El trabajo se ha estructurado de la siguiente forma. Tras esta introducción, el apartado segundo se dedica al estudio del contexto teórico que nos permite sustentar nuestras hipótesis. El apartado tercero se dedica a la descripción de las hipótesis que pretenden someterse a contraste empírico. En el apartado cuarto se especifica el modelo que pretende estimarse y se describe la muestra. En el quinto apartado se describen los resultados obtenidos en nuestro estudio. El sexto apartado trata de analizar la robustez 
de los resultados a distintas especificaciones de variables relevantes y a la diferente composición de la muestra. El último apartado pretende resaltar las principales conclusiones alcanzadas en nuestro estudio, así como las limitaciones del mismo.

\section{EL ACCESO DE LA MUJER AL CONSEJO DE ADMINISTRACIÓN Y LA CULTURA DEL PAIS}

El consejo de administración, como órgano de gobierno de la empresa, supervisa y controla la gestión de la empresa y diseña las estrategias a llevar a cabo por ésta. Numerosos estudios han venido resaltando que para que una empresa tenga éxito en el desarrollo de su actividad, requiere un eficiente y eficaz consejo de administración que lleve a cabo sus funciones con integridad y competencia. En este sentido, gran parte de la literatura sobre consejos de administración, hace descansar su eficacia de funcionamiento en aspectos tales como la composición del mismo y la independencia de sus miembros (Baysinger y Butler, 1985; Weisbach, 1988; Hermalin y Weisbach, 1988, 1991; Rosenstein y Wyatt, 1990, 1997; Bhagat y Black, 2000). Como extensión a la literatura que somete a estudio la composición de los consejos de administración y sobre la naturaleza de los miembros que componen estos consejos, es donde cabe situar el tema de la representación de la mujer en los consejos de administración.

La evidencia empírica de la que se dispone revela que existen diferencias entre el acceso de la mujer a los consejos de administración cuando se comparan distintos países. Al objeto de comprender cuáles son las posibles razones que pueden explicar el diferente nivel de acceso de la mujer a los consejos de administración cuando se comparan países distintos, diversos estudios señalan como las diferencias en el ámbito institucional son la base para las divergencias en las estructuras de gobierno a nivel internacional (Aguilera y Jackson, 2003; Lubatkin et al., 2005 y 2007; Li y Harrison, 2008). Estos estudios analizan la influencia del contexto institucional sobre los mecanismos de gobierno corporativo, reconociendo la importancia del papel de las diferencias institucionales, como son los aspectos legales o culturales, en las prácticas de gobierno de las empresas.

La teoría institucional asume que las organizaciones son cautivas del entorno institucional en el que se encuentran (Meyer y Rowan, 1977). En orden a sobrevivir en entornos competitivos y adquirir legitimidad, las organizaciones deben acomodar su estructura organizativa al conjunto de normas y sistemas de creencias que prevalecen en su entorno inmediato. El pilar normativo en el que descansa toda sociedad, envuelven un conjunto de obligaciones normativas, así como valores y creencias compartidas por todos los miembros de la sociedad que determinan el comportamiento social esperado (DiMaggio y Powell, 1983; Scott, 1987). De esta forma, la conformidad con las expectativas sociales son consideradas un elemento clave para la supervivencia de la empresa. Las empresas son por tanto entidades sociales integradas en un orden institucional establecido, por lo que las empresas, en orden a adquirir legitimidad, tenderán a conformar sus estructuras organizativas al orden social establecido. Dado que la cultura, como conjunto de creencias y valores, es una de las fuerzas institucionales de toda sociedad, la forma en la que queda configurada la estructura de las organizaciones puede estar fuertemente condicionada por los valores culturales que 
prevalecen en una sociedad dada. En este sentido, trabajos pioneros como los de Friedland y Alford (1990), Hofstede (1991) y Hickson y Pugh (1995) concluyen que las características culturales de un pais, como rasgos del entorno institucional del mismo, tienen una gran influencia sobre la estructura de gobierno de las empresas.

La cultura está formada por un conjunto de ideas que han venido seleccionándose históricamente, ideas éstas que conllevan una serie de valores que tienen capacidad para explicar el comportamiento de los individuos en la sociedad. No obstante, tal como señalábamos anteriormente, la cultura puede ser sometida a análisis desde distintos niveles adquiriendo significado y relevancia el concepto de cultura de un país. Para Hofstede (1980) la cultura de un país puede ser considerada como la programación social colectiva que determina el conjunto de valores, creencias, convicciones y actitudes que comparten los miembros de un colectivo social determinado. Esta cultura, tal como sugieren House et al. (2004), sirve como marco de referencia que posibilita interpretar y dar significado a los eventos significativos que resultan de experiencias comunes de los miembros de un colectivo, los cuales, cuestión de gran importancia, son trasmitidos a través de generaciones. El rasgo fundamental de la cultura es que ésta supone un diseño social que condiciona la mayoría de las prácticas y procesos sociales, por lo que gran parte de los comportamiento sociales pueden ser comprendidos a través de la cultura prevaleciente.

Una importante cuestión en relación a la cultura, como han revelado numerosos estudios (Hofstede, 1980; Schwartz, 1992, 1994; Ingleharts, 1977, 2001, 2004; Trompenaars, 1993; House et al., 2004), es la existencia de diversidad cultural entre países, lo cual se materializa en que sociedades distintas responden de manera diferente ante los mismos fenómenos. En este sentido, al objeto de este estudio, utilizaremos el marco cultural propuesto por Hofstede $(1980 ; 1991 ; 2000)$, por ser uno de los que más influencia ha tenido en la comparación cultural entre países ${ }^{(2)}$. El modelo cultural de Hofstede parte de la asunción de que la cultura de un país es observable y determinable empíricamente. Para ello este autor derivó y definió cuatro dimensiones de valoración cultural, las cuales se basan en cuatro problemas centrales de toda sociedad como son la relación entre el individuo y el grupo, la desigualdad social, las implicaciones sociales del género y la forma de tratar la incertidumbre inherente a todo proceso social. El objetivo de su estudio era determinar si los miembros de un país establecían pautas de comportamiento similares en relación a estas cuestiones, y que resultaran diferenciables de las de los miembros de otros países. El análisis empírico original de Hofstede (1980) se basó en un cuestionario dirigido a los empleados de IBM en 40 países y en dos períodos de tiempo (1967-1968) y (1971-1973). La respuestas realizadas por los sujetos entrevistados, y la posterior agregación de los valores atendiendo al país de procedencia de los mismos, permitió a Hofstede la identificación de cuatro dimensiones culturales que distinguían a los distintos países y que constituyen

(2) Múltiples estudios han resaltado que los valores culturales, utilizando fundamentalmente el trabajo seminal de Hofstede (1980), influyen en diversas decisiones de gestión en las empresas (entre ellos, Gray, 1988; Ralston et al., 1993; Salter y Niswander, 1995; Pendersen y Thomsen, 1997; Semenov, 2000; Stulz y Williamson, 2003; Hope, 2003; House et al., 2004; Guiso et al., 2006). 
los aspectos determinantes de la cultura del país ${ }^{(3)}$. Dichas dimensiones fueron denominadas como distancia de poder, aversión a la incertidumbre, individualismo y masculinidad $^{(4)}$. En este sentido, siendo ésta la principal contribución del modelo cultural de Hofstede, la identificación y evaluación de estas dimensiones culturales posibilita el análisis comparativo entre países y en particular como afecta la cultura del país a diversos fenómenos sociales ${ }^{(5)}$.

En lo que respecta al objeto de este estudio, la cultura de un país puede determinar una asignación social de roles distintos para el hombre y la mujer, es decir, puede crear un estereotipo de género. En si mismo el concepto de género es un concepto culturalmente creado para hacer referencia a las diferencias entre hombres y mujeres en la sociedad en relación a actitudes, estructuras mentales y expectativas, las cuales trascienden a las diferencias biológicas. Concepto como igualdad de género o discriminación de género, constituyen una manifestación de las creencias sociales sobre la apropiada distribución de papeles entre hombre y mujer. Unas sociedades parecen minimizar estas diferencias en la asignación de roles, mientras que otras por el contrario las maximizan (House et al., 1999). Basándonos en este argumento el objetivo de este trabajo es analizar si el mayor o menor nivel de representación de la mujer en los consejos de administración de empresas situadas geográficamente en países distintos puede venir explicada por la cultura del país.

\section{FORMULACIÓN DE HIPÓTESIS}

Como hemos venido resaltando el objetivo de nuestro estudio es analizar si la cultura que prevalece en un país determinado puede explicar el nivel de acceso de la mujer al consejo de administración cuando se comparan países distintos. En este sentido, en este epígrafe pretendemos analizar la posible relación entre las dimensiones culturales de Hofstede y el acceso de la mujer al consejo de administración, al objeto de plantear un conjunto de hipótesis que puedan ser contrastadas empíricamente.

Como señalamos anteriormente Hofstede identificó cuatro dimensiones culturales que son capaces de identificar la singularidad cultural de los distintos países, siendo estas dimensiones: distancia de poder, aversión a la incertidumbre, individualismo y masculi$\operatorname{nidad}^{(6)}$.

(3) Posteriormente Hofstede añadió una quinta dimensión cultural denominada orientación a largo plazo, si bien, al no ser ésta evaluada para todos los países no ha sido objeto de atención en la mayoría de los estudios que pretenden realizar comparaciones entre la cultura de distintos países.

(4) Con posterioridad procederemos a la definición estricta del significado de estas dimensiones culturales, las cuales constituyen la básica de la métrica utilizada en la caracterización de los factores determinantes de la cultura de un país que será utilizada en este estudio.

(5) Una de las principales críticas que se le ha venido haciendo al modelo cultural de Hofstede es que presenta una visión sobresocializada en el sentido de que se asume que todos los miembros de un país están completamente conformes con los valores y pautas que determinan las distintas dimensiones culturales identificadas. Obviamente resulta poco probable que todos los individuos de una sociedad dada reflejen de forma homogénea los valores culturales de dicha sociedad, si bien, ello no invalida los estudios comparativos entre países.

(6) Posteriormente Hofstede en 1987 añadió una quinta dimensión cultural, orientación a largo plazo la cual pretende captar la orientación de largo plazo de los miembros de una sociedad, es decir, el grado en el que los miembros de la sociedad aceptan postergar la gratificación de sus necesidades materiales, sociales y emocionales. Un valor bajo indica cultura donde los cambios pueden ocurrir más rápidamente, debido a que los compromisos de largo plazo no son impedimentos de cambio (Hofstede 2000). No obstante, esta dimensión cultural no será objeto de atención en este estudio. 
La dimensión cultural distancia de poder es definida como el grado en el que una sociedad acepta la distribución desigual de poder en organizaciones e instituciones. Un valor alto de esta dimensión cultural indica que las desigualdades de poder y riqueza son consideradas aceptables dentro de la sociedad, es decir, los miembros menos poderosos aceptan como algo natural que el poder esté distribuido desigualmente. Esta dimensión puede tener múltiples implicaciones en relación a cuestiones diversas como la importancia concedida al estatus, poder y prestigio, si bien, al objeto de nuestro estudio resaltaremos el efecto que puede tener en los procesos sociales para el cambio en la distribución de poder en la sociedad. En efecto, al aceptarse la distribución desigual de poder como algo natural, los miembros de la sociedad tienden a aceptar su posición en el sistema social en países de alta distancia al poder. Dado que como resulta contrastable empíricamente la mujer participa menos que los hombres en la estructura de poder en las organizaciones, la mujer puede considerar esto como un orden de desigualdad legítimo, lo que puede explicar, tal como sugieren diversos estudios (Daily y Dalton, 1995; Hillman et al, 2002; Singh et al., 2002; Farrell y Hersch, 2005), el propio rechazo de las mujeres para autopromocionarse dentro de las organizaciones o para dirigir activamente su carrera para promocionar en la empresa. Basándonos en este argumento se esperaría que en aquellas sociedades que manifestaran un mayor valor para la distancia al poder sería menor el nivel de representación de mujeres en el consejo de administración de las empresas, por lo que esperamos que la relación entre ambas variables sea negativa. Siguiendo este razonamiento la hipótesis que formulamos es la siguiente:

H1. La representación de la mujer en los Consejos de Administración será más baja en aquellos países donde el valor en la dimensión cultural distancia en el poder es mayor.

La dimensión cultural aversión a la incertidumbre hace referencia a la respuesta de una sociedad frente a contextos ambiguos y no estructurados. En culturas con una alta aversión a la incertidumbre los miembros de la sociedad basan su comportamiento en procedimientos claros, estrategias bien conocidas y normas bien comprendidas para reducir la incertidumbre y hacer frente a situaciones desconocidas. Por el contrario, en culturas con baja aversión a la incertidumbre hay una mayor tolerancia hacia la incertidumbre y hacia ideas, aproximaciones y conceptos diferentes. En este sentido, tal como han resaltado diferentes autores (Schwartz y Harrison, 1986; Burke, 1997; Zelechowski y Bilimoria, 2004), las mujeres tienen en relación a los hombres diferentes experiencias en el trabajo, mercado, servicios públicos y la comunidad, por lo que las mujres aportan una diferente perspectiva en el debate y la toma de decisiones. Por esta razón, resulta probable que en culturas con una menor aversión a la incertidumbre se acepte con mayor facilidad nuevas aproximaciones e ideas en el seno de los consejos de administración. Por lo tanto la asociación esperada entre la dimensión cultural aversión a la incertidumbre y el acceso de la mujer al consejo de administración de las empresas sería negativa. La hipótesis que formulamos es la siguiente: 
H2. La representación de la mujer en los Consejos de Administración será más baja en aquellos países donde el valor en la dimensión cultural aversión a la incertidumbre es mayor.

La dimensión cultural individualismo hace referencia a la relación que existe entre los individuos y los grupos sociales más amplios a los que pertenecen. En las culturas individualistas prevalecen la conciencia y el interés individual, mientras que en las culturas colectivas el interés del colectivo prevalece sobre los intereses individuales. Se trata por tanto de una medida del grado en el cual una sociedad valora los objetivos personales, autonomía y privacidad sobre la lealtad al grupo, el compromiso con las normas grupales, actividades colectivas y cohesión social. En este sentido, en sociedades colectivas el individuo se define y adquiere interés en relación al grupo. Dado que el interés agregado tiende a prevalecer sobre el interés de los individuos, conceptos como bienestar de grupos y compromiso son prominentes en sociedades colectivas. De esta forma, en las sociedades colectivas suele existir mayor sensibilidad hacia la representación de las minorías en los órganos de decisión de la sociedad (Very et al, 1997; Schuler y Rogovsky, 1998), por lo que en éstos están representados por el más diverso rango de grupos sociales, lo cual puede explicar que en los consejos de administración puedan estar representadas un mayor número de mujeres. Por esta razón esperamos una relación negativa entre la dimensión cultural individualismo y el acceso de la mujer a los consejos de administración. En términos formales emitimos la siguiente hipótesis:

H3. La representación de la mujer en los Consejos de Administración será más baja en aquellos países donde el valor en la dimensión cultural individualismo es mayor.

Por último, la dimensión cultural masculinidad es el grado en el cual valores como asertividad, desempeño, éxito y competitividad, asociados con el rol masculino, prevalecen sobre valores como la calidad de vida, relaciones personales, servicio, solidaridad, valores estos últimos asociados con el rol femenino. En este sentido, las sociedades masculinas definen los roles de género de forma tradicional y esteriotipados, teniendo visiones más cerradas sobre la diversidad de papeles que hombres y mujeres pueden desempeñar dentro de la organización social. En este sentido, Konrad et al. (2000) y Oakley (2000) muestran evidencia empírica que revela que la atribución de valores estereotipados hacia las mujeres puede sesgar la elección de éstas en los consejos de administración de las empresas. Valores altos de la dimensión cultural masculinidad indican que los valores asociados al rol masculino prevalecen en la sociedad, motivo por el cual esperamos un signo negativo en la relación que liga los valores que alcancen esta dimensión cultural y el acceso de la mujer a los consejos de administración. Basándonos en este argumento emitimos la siguiente hipótesis:

H4. La representación de la mujer en los Consejos de Administración será más baja en aquellos países donde el valor en la dimensión cultural masculinidad es mayor. 


\section{ESTUDIO EMPÍRICO}

\subsection{MOdelo Y VARIABLES}

Al objeto de analizar en que medida el acceso de la mujer a los consejos de administración de las empresas puede estar afectada por la cultura que prevalece en un país, estimaremos un modelo en el que las variables experimentales serán las dimensiones culturales identificadas por Hofstede (1980). Para controlar las diferencias inter-empresas que pueden explicar distintos niveles de acceso de la mujer a los consejos de administración de las empresas, con independencia del país de origen de las mismas, introducimos una serie de variables de control. En términos analíticos el modelo puede quedar especificado en los siguientes términos:

$$
\begin{gathered}
\text { PARTICIPACIÓN }=\beta_{1}+\beta_{2} \text { DIPODER }+\beta_{3} \text { INCERTIDUMBRE }+\beta_{4} \text { INDIVIDUALISMO + } \\
\beta_{5} \text { MASCULINIDAD }+\beta_{6} \text { ACTIVIDAD }+\beta_{7} \text { CONSEJEROS }+\beta_{8} \text { RENTABILIDAD }+ \\
\text { B9CÓDIGO }+\sum_{j=1}^{10} \text { SECTORj }+\xi
\end{gathered}
$$

La variable dependiente, denotada como PARTICIPACIÓN, pretende evaluar el acceso de la mujer a los puestos de representación del consejo de administración de la empresa. Esta variable pretende medir una dimensión cuantitativa del nivel de acceso de la mujer a los consejos de administración y ha sido calculada a través del cociente entre el número de mujeres representadas en dichos consejos, dividido por el número total de miembros del consejo.

Respecto a las variables experimentales utilizadas para captar el efecto cultura de los distintos países representados en la muestra objeto de estudio, tomaremos las variables y los valores atribuidos a las diferentes dimensiones culturales dadas por Hofstede (1980) para los distintos países. Distancia de poder, denotada en este trabajo como DIPODER, aversión a la incertidumbre denominada INCERTIDUMBRE en este estudio, individualismo, denominada INDIVIDUALISMO y, por último masculinidad, denotada por MASCULINIDAD ${ }^{(7)}$.

Aun cuando los resultados obtenidos en investigaciones previas no resultan concluyentes, en primer lugar vamos a considerar el posible efecto que tiene el tamaño de las empresas sobre el nivel de participación de la mujer en el consejo de administración. En relación a la relación que liga el tamaño de las empresas y el nivel de participación de la mujer, algunos estudios previos han encontrado que en empresas pequeñas la mujer tienen mayores posibilidades de promocionar en la estructura orgánica de la empresa (Bertrand y Hallock, 2001; Andre, 1995), debido fundamentalmente a que plantillas reducidas posibilitan un mejor conocimiento de la capacidad potencial de la mujer para desarrollar las funciones encomendadas a los miembros del consejo de administración. Por el contrario, otros estudios sobre el nivel de representación de la mujer en los consejos de administración encuentran una relación positiva entre el tamaño de la empresa y la presencia de la mujer en los consejos (Harrigan, 1981; Heidrick

(7) En un epígrafe posterior se aportaran los valores que adquieren estas dimensiones en los distintos países objeto de nuestro estudio. 
y Struggles, 1977; Catalyst, 2001), aspecto éste que podría quedar explicado porque la existencia de procesos formalizados de evaluación y promoción en grandes firmas posibilita que el acceso de la mujer a los puestos de responsabilidad venga determinado por su formación y capacidad. Por esta razón, en este estudio no se predetermina el signo esperado para la relación que liga el nivel de actividad y la representación de la mujer en los consejos de administración. Al objeto de cuantificar esta variable, numerosas investigaciones han utilizando la cifra de negocios (Hillman y Cannella, 2007; Hambrick y Cannella, 2004; Sander y Boivie, 2004; Coffey y Wang (1998); McCormick Hyland y Marcellino, 2002), el total activo (Peng, 2004; Carter et al., 2003; Erhardt, et al., 2003) o el número de empleados (Konrad y Mangel, 2000; Coffey y Wang, 1998; y Smith, 2007). En nuestro estudio vamos a utilizar como medio de inferir el tamaño de la empresa la variable ACTIVIDAD medida, al objeto de evitar posibles problemas de escala, a través del logaritmo del nivel de facturación de las empresas.

Para analizar si el propio tamaño del consejo de administración puede afectar al nivel de representación de las mujeres en el mismo, introducimos la variable CONSEJEROS calculada a través del número total de consejeros que forman el consejo de administración (Coffey y Wang, 1998; Carter, et al., 2003; y Erhardt, et al., 2003; Hillman y Cannella, 2007). Se espera que mientras mayor sea el tamaño del consejo, mayor será la oportunidad de la mujer para acceder al mismo, motivo por el cual esperamos una relación positiva entre el tamaño del consejo de administración y el porcentaje de mujeres representadas en dichos consejos.

Estudios previos (como el de Litz y Folker, 2002) relacionan liderazgo participativo, trabajo en equipo y orientación al cliente, factores que presuponen rendimientos superiores, con una mayor presencia de mujeres en los consejos de administración. Para analizar en que medida el rendimiento diferencial de una empresa puede justificar un mayor acceso de la mujer a los puestos del consejo de administración, hemos introducido en nuestro modelo la variable de control RENTABILIDAD calculada como el beneficio neto dividido entre el total de los fondos propios (Adler, 2001; Carter et al., 2003; Catalyst, 2004).

Adicionalmente, queremos controlar los esfuerzos institucionales realizados en los distintos países para promover la participación de la mujer en los consejos de administración. En efecto, determinados gobiernos están propiciando una política de paridad, preocupándose, en mayor o menor medida, de introducir en los Códigos de Buen Gobierno temas relativos a la diversidad en los consejos de administración, y en concreto algunos de ellos recomiendan que se ponga especial atención en establecer un equilibrio entre miembros hombres y miembros mujeres en el consejo (Noruega, Finlandia, Suecia y España) ${ }^{(8)}$. En este sentido, se crea la variable dicotómica CóDIGO,

(8) En este sentido, el Gobierno Noruego ha realizado una apuesta fuerte a favor de la diversidad de género en los consejos, ya que desde enero de 2004 las empresas públicas tienen que tener una representación de cada sexo de al menos el $40 \%$ del consejo de administración; en cuanto al sector privado, ha tenido un plazo de adaptación hasta julio de 2005, con penalizaciones en 2007 en caso de que las empresas no cumplan con lo previsto. En 2008 el promedio de mujeres que formaban parte de los consejos era del 44\%, el mayor porcentaje de todos los países europeos, mientras que en 2004 el porcentaje era del 22\% (EPWM, 2004-2008). El Código Unificado Español (CNMV, 2006), dedica el apartado 15 a la diversidad, y, recomienda que el consejo refleje la diversidad de conocimientos, de género y de experiencias precisas para desempeñar sus 
la cual toma valor 1 cuando los códigos de buen gobierno de los países sometidos a estudio hacen referencia a la necesidad de incorporar mujeres en los consejos (Suecia, España e Inglaterra), y 0 cuando tal referencia no se realiza (Bélgica y Dinamarca) ${ }^{(9)}$.

Por último, al objeto de analizar si las diferencias en el acceso de la mujer a los consejos de administración vienen explicadas por factores distintos a las dimensiones culturales que caracterizan a una sociedad, hemos introducido en el modelo otras variables de control que recojan las diferencias entre las distintas empresas objeto de nuestro análisis con independencia del país de origen de las mismas. La selección de estas variables viene justificada porque han adquirido capacidad explicativa en diversos estudios que han intentado explicar el acceso de la mujer a los puestos del consejo de administración de las empresas. En primer lugar, dado que, como indican Brewer (2001) y McDonals (2000), el sector de actividad en el que opera la empresa, dada la distinta tradición y dinámica de los mismos, afecta a las oportunidades que tiene la mujer para acceder a niveles de responsabilidad, hemos clasificado las empresas en distintos sectores en los que desarrollan su actividad. En este sentido, las distintas observaciones han sido clasificadas atendiendo al sector de actividad en el que las empresas operan, distinguiéndose los siguientes sectores: Energía; Materiales primarios, Industria, Bienes de Consumo, Salud, Servicio, Sector Público, Telecomunicaciones, Financiero, Tecnológico.

\subsection{Muestra}

La muestra objeto de este estudio está compuesta por distintas empresas de diversos países, en concreto de Bélgica, Dinamarca, España, Inglaterra y Suecia. La selección de estos países viene motivada por diversas razones. En primer lugar, estos países, según demuestran Carrasco y Laffarga (2008), son los que dentro del contexto europeo muestran diferencias más significativas en cuanto a porcentaje de mujeres en los consejos de administración. En segundo lugar, son países clasificados tradicionalmente en sistemas jurídicos diferentes como son el sistema Anglosajón o de ley común (Inglaterra), el sistema Escandinavo o ley civil de origen escandinavo (Dinamarca y Suecia) y el sistema mediterráneo o ley civil de origen Francés (Bélgica y España), aspectos éstos que pueden implicar diferente regulación en torno a la paridad por razón de género de los países. Una vez determinados los diversos países que mantienen tradiciones culturales diferentes y diferente nivel de representación de la mujer en los consejos de administración, hemos utilizado la Base Internacional Osiris en la cual aparecen representadas empresas de los distintos países objeto de estudio. En principio fueron seleccionadas todas las empresas de los distintos países recogidas en dicha base de datos. No obstante,

funciones con eficacia, objetividad e independencia e invita a las sociedades con escasa presencia femenina en sus Consejos a que hagan un esfuerzo deliberado por buscar posibles candidatas cada vez que deba cubrirse alguna vacante en el Consejo, especialmente para puestos de independientes. En el mismo sentido se mueve el artículo 75 de la Ley Orgánica de Igualdad (2007), que pretende alcanzar una presencia equilibrada de mujeres y hombres en el plazo de ocho años. El porcentaje de mujeres en los consejos de administración en España ha pasado del 3\% al 6,6\% de 2004 a 2008 (EPWN, 2004-2008).

(9) Para asignar valor a esta variable, teniendo en cuenta la fecha de los datos utilizados, hemos tomado las recomendaciones de los códigos de buen gobierno aprobados hasta 2006. En 2008 y 2009 se han revisado los códigos Danés y Belga, recomendando la diversidad de género en los consejos de administración. 
se realizó un ejercicio de depuración para que la información contable fuera homogénea. A tal fin los datos recogidos en Osiris se acotaron en función de la información contable consolidada de las empresas cotizadas relativas al ejercicio 2.006, elaborada con base en las normas internacionales de contabilidad. Por necesidades operativas se establecieron algunas restricciones, en particular para el caso de Inglaterra, dado que el alto volumen de empresas representadas en dicha base hacía que no resultara factible el tratamiento completo de las empresas registradas. Por esta razón se optó por estratificar a la muestra de empresas inglesas en función del número de empleados, de tal forma que éstos se dividieron en cuartiles; de cada cuartil sólo se trabajó con las primeras cien empresas que poseían mayor número de empleados. Un total de 400 empresas fueron seleccionadas, número que disminuyó a 213 al limitarnos sólo a aquellas empresas que presentaban información consolidada conforme a las NIIF. En cuanto a España, además de la información contable para el ejercicio 2006 elaborada con base en las normas internacionales, se seleccionó como criterio adicional que las empresas estuvieran registradas en la Comisión Nacional del Mercado de Valores (CNMV). Adicionalmente hemos utilizado la información contenida en los informes de buen gobierno del año 2006 de las empresas cotizadas para completar la base.

La muestra definitiva está compuesta por 989 empresas de cinco países europeos. 131, españolas; 213, inglesas; 117, belgas; 150 , danesas; 378 , suecas.

\section{RESULTADOS}

\subsection{EsTADíSTICOS DESCRIPTIVOS}

En la tabla 1 se recogen algunos datos que caracterizan los datos muestrales con los que se aborda este estudio ${ }^{(10)}$.

TABLA 1

EsTadísTICOS DESCRIPTIVOS

\begin{tabular}{|l|c|c|c|c|}
\hline \multicolumn{1}{|c|}{ Variable } & Mínimo & Máximo & Media & Desviación típica \\
\hline PARTICIPACIÓN & 0 & 0,70 & 0,088 & 0,11 \\
\hline CONSEJEROS & 1 & 26 & 7,86 & 3,43 \\
\hline ACTIVIDAD & 0 & 319056000 & 4679594,3 & 21457638,462 \\
\hline RENTABILIDAD & -775 & 298 & 9,38 & 57,753 \\
\hline
\end{tabular}

Como se puede observar en la tabla 1, el valor medio de la variable PARTICIPACIÓN queda situado en torno al nueve por ciento, dato este consistente con investigaciones previas que revelan el escaso nivel de representación de las mujeres en los niveles más altos de la cúpula de dirección y control de las empresas.

(10) En estas estadísticas descriptivas reproducimos, en lo que se refiere a la variable actividad, los importes sin la transformación en logaritmo de la misma. 
En la tabla 2 se recogen las correlaciones entre las distintas variables, dependiente, experimentales y de control, que serán estimadas según el modelo descrito en el apartado 3.1. Tal como puede observarse en la tabla, las correlaciones entre la variable PARTICIPACIÓN y las dimensiones culturales siempre es negativa, siendo significativa en tres de las dimensiones objeto de nuestra atención (DIPODER, INCERTIDUMBRE y MASCULINIDAD) ${ }^{(11)}$.

\section{TABLA 2}

MATRIZ DE CORRELACIONES

\begin{tabular}{|c|c|c|c|c|c|c|c|c|}
\hline Variables & PARTICIPACIÓN & CONSEJEROS & ACTIVIDAD & RENTABILIDAD & DIPODER & INCERTIDUMBRE & INDIVIDUALISMO & MASCULINIDAD \\
\hline PARTICIPACIÓN & 1.000 & $0.076^{\circ}$ & $0.113^{\prime \prime}$ & 0.016 & $-0.137^{\circ}$ & $-0.173^{\prime \prime}$ & -0.049 & $-0.268^{* \prime}$ \\
\hline CONSEJEROS & & 1.000 & $0.244^{\prime \prime}$ & 0.011 & $0.280^{\circ \prime}$ & $0.291^{\prime \prime}$ & -0.056 & $0.248^{\circ *}$ \\
\hline ACTIVIDAD & & & 1.000 & $0.082^{\circ}$ & -0.060 & $-0.066^{\circ}$ & 0.020 & -0.040 \\
\hline RENTABILIDAD & & & & 1.000 & $0.090^{\circ \prime}$ & $0.116^{\prime \prime}$ & -0.016 & 0.134 \\
\hline DIPODER & & & & & 1.000 & $0.968^{\circ \prime}$ & $0.351^{* *}$ & $0.526^{* *}$ \\
\hline INCERTIDUMBRE & & & & & & 1.000 & $0.457^{* \prime}$ & $0.493^{\circ *}$ \\
\hline INDIVIDUALISMO & & & & & & & 1.000 & $0.400^{\circ}$ \\
\hline MASCULINIDAD & & & & & & & & 1.000 \\
\hline
\end{tabular}

** Indica que la correlación es significativa al nivel 0,01 (bilateral).

* Indica que la correlación es significativa al nivel 0,05 (bilateral).

TABLA 3

VALORES MEDIO DE LA PRESENCIA DE MUJERES EN EL CONSEJO DE ADMINISTRACIÓN Y TAMAÑO DEL CONSEJO

\begin{tabular}{|c|c|c|c|c|c|c|}
\hline Variable & Bélgica & Dinamarca & España & Inglaterra & Suecia & Test ( $p$-value) \\
\hline \multicolumn{7}{|c|}{ Panel A : Porcentaje de mujeres en los consejos de administración por países } \\
\hline PARTICIPACIÓN & 0,050 & 0,069 & 0,065 & 0,055 & 0,134 & $\begin{array}{c}112,377 \\
(0,000)\end{array}$ \\
\hline \multicolumn{7}{|c|}{ Panel B : Valores de las dimensiones culturales } \\
\hline $\begin{array}{l}\text { DIPODER } \\
\text { INCERTIDUMBRE } \\
\text { INDIVIDUALISMO } \\
\text { MASCULINIDAD }\end{array}$ & $\begin{array}{l}65 \\
94 \\
75 \\
54\end{array}$ & $\begin{array}{l}18 \\
23 \\
74 \\
16\end{array}$ & $\begin{array}{l}57 \\
86 \\
51 \\
42\end{array}$ & $\begin{array}{l}35 \\
35 \\
89 \\
66\end{array}$ & $\begin{array}{c}31 \\
29 \\
71 \\
5\end{array}$ & \\
\hline \multicolumn{7}{|c|}{ Panel C : Tamaño medio del consejo de administración por países } \\
\hline CONSEJEROS & 8,70 & 7,05 & 10,82 & 7,62 & 7,02 & $\begin{array}{l}100,618 \\
(0,000)\end{array}$ \\
\hline
\end{tabular}

\subsection{ANÁlisis UNIVARIANTE DE LA DIFERENCIA DE ACCESO DE LAS MUJERES AL CONSEJO} DE ADMINISTRACIÓN ENTRE PAÍSES

Dado que nuestro estudio tiene por objeto analizar si existen diferencias en el acceso de la mujer a los consejos de administración entre distintos países, en lo que sigue

(11) De las 36 correlaciones posibles entre las variables, en 18 ocasiones estas correlaciones son significativas, lo que hace recomendable tratar el problema de multicolinealidad que pueden provocar las mismas. 
describiremos una serie de datos al objeto de profundizar en el conocimiento del distinto nivel de representación de la mujer en los consejos de administración atendiendo al país de origen de las distintas empresas representadas en nuestra muestra. En primer lugar en la tabla 3, Panel A, se recogen los valores medios que alcanzan en los distintos países la variable PARTICIPACIÓN, junto al estadístico no paramétrico de Kruskal-Walis para determinar si existen diferencias estadísticas significativas entre los distintos países.

Como puede observarse en los resultados recogidos en el panel A de la tabla 3, la variable PARTICIPACIÓN muestra diferencias significativas entre los países atendiendo al valor del estadístico utilizado. Profundizando en los datos, Suecia es el país que mayor nivel de presencia de mujeres tiene en los consejos de administración con aproximadamente un 14 por ciento, seguido de Dinamarca por aproximadamente un siete por ciento, y por España con 6,5 por ciento. Inglaterra tiene un menor nivel de representación de mujeres según los datos de nuestra muestra con un 5,5 por ciento, seguido de Bélgica con un cinco por ciento. En suma, el nivel de representación de la mujer en los consejos de administración es distinto dependiendo de la procedencia geográfica de las empresas de la muestra.

En el panel B de la tabla 3 se recogen los valores que alcanzan las diferentes dimensiones culturales que son utilizadas en nuestro estudio en los distintos países. Sobre estas no realizamos comentario alguno en la medida que la propia literatura las considera como dimensiones sobre las que se constituyen las diferencias culturales entre países.

En el panel C de la tabla 3 se recogen los valores medios del tamaño del consejo de administración por países, dado que puede resultar interesante analizar en que medida la mayor o menor representación de la mujer puede venir explicada por la propia dimensión del consejo de administración. Como podemos observar las diferencias en el tamaño del consejo de administración entre los distintos países es estadísticamente significativa. El menor tamaño de los consejos se encuentra en Suecia, mientras que la representación en términos porcentuales es la mayor de los diversos países analizados; es decir, el mayor nivel de representación de mujeres se alcanza para consejos de menor tamaño en términos absolutos, lo que convierte a los mismos en consejos más equilibrados atendiendo a la variable género. En el extremo opuesto se encuentra el caso español, donde el nivel de representación de mujeres se alcanza para tamaños amplios de los consejos de administración, lo que puede implicar que el mayor número de mujeres representadas se ha ido obteniendo no a través de políticas de equilibrio entre mujeres y hombres, sino a través de incrementar el número de miembros del consejo de administración.

Para profundizar más en la dinámica de acceso de la mujer a los consejos de administración, en la tabla 4 se analiza tanto el número de mujeres que han accedido a los consejos de administración de las empresas que forman parte de nuestra muestra, como el tamaño medio de los consejos en los que existe representación de la mujer. 
TABLA 4

NÚMERO DE MUJERES EN LOS CONSEJOS DE ADMINISTRACIÓN Y TAMAÑO MEDIO DEL CONSEJO

\begin{tabular}{|c|c|c|c|c|}
\hline \multirow{2}{*}{\begin{tabular}{c} 
Número de mujeres \\
\cline { 2 - 5 }
\end{tabular}} & \multicolumn{2}{|c|}{ Número de empresas } & \multicolumn{2}{c|}{ Tamaño del Consejo } \\
\cline { 2 - 5 } & 500 & $\%$ & Valor medio & $\%$ mujeres en el consejo \\
\hline 0 & 330 & 33,4 & 9,99 & 0,11 \\
\hline 1 & 109 & 11,0 & 10,21 & 0,19 \\
\hline 2 & 32 & 3,2 & 13,23 & 0,23 \\
\hline 3 & 14 & 1,4 & 12,72 & 0,31 \\
\hline 4 & 2 & 0,2 & 12,50 & 0,40 \\
\hline 5 & 2 & 0,2 & 13,00 & 0,54 \\
\hline
\end{tabular}

En la columna izquierda de la tabla 4 puede analizarse el distinto número de mujeres que están representadas en los consejos de administración Del total de observaciones que componen nuestra muestra, en 500 casos, es decir, en un 50,6 por ciento de los casos, no hay presencia de mujeres en los consejos de administración, mientras que en 489 casos, lo que representa un 49,4 por ciento, al menos existe una mujer en el consejo de administración. Este resultado es quizás más revelador de la dificultad de la mujer para acceder a los puestos del consejo de administración, dado que en aproximadamente un 50 por ciento de los consejos de administración no existe presencia alguna de mujeres, revelándose por tanto que en un elevado porcentaje los puestos de consejeros todavía quedan reservados para hombres. Igualmente es destacable el alto porcentaje de consejos en lo que sólo existe una mujer $(33,4 \%)$, lo que revela una tímida presencia de la mujer, aspecto éste que podría venir explicado más por respuesta de las empresas a las presiones institucionales de diversidad e igualdad de representación, que por una plena convicción del importante papel que puede jugar la mujer en estos órganos de control empresarial. Como también puede observarse el número de consejos donde existen tres o más mujeres, se reduce de manera significativa (aproximadamente un cinco por ciento).

Los anteriores datos son suficientemente reveladores de las importantes trabas que sufren las mujeres para acceder a los consejos de administración, aun cuando estas trabas pueden resultar distintas dependiendo del país de origen de las empresas. Para aportar evidencia empírica sobre esta cuestión, hemos procedido a analizar el número de consejos donde existe presencia de mujeres clasificada en relación a los distintos países que son objeto de análisis en este trabajo, datos estos que no han sido tabulados. Un análisis individualizado pone de manifiesto que Suecia es el único país donde existen más consejos de administración en los que la presencia de mujeres es mayor a los que no existe presencia de éstas. En un segundo nivel se encuentran los casos de Dinamarca y España donde aun siendo mayor el porcentaje de consejos donde no está representada la mujer, los porcentajes están cercanos al 50 por ciento. Los casos de Bélgica e Inglaterra determinarían el tercer nivel, donde aproximadamente en un 65 por ciento de consejos no existe representación de mujeres, lo que implica que son en 
estos países donde las dificultades de acceso de la mujer son mayores. Estos datos, aun dada su naturaleza descriptiva y univariante, avalan nuestra tesis de que la cultura del país puede convertirse en un factor determinante a la hora de explicar el acceso de la mujer a los puestos de los consejos de administración de las empresas.

Otra cuestión importante a analizar es el tamaño medio de los consejos en función del distinto número de mujeres presente en el mismo, dado que ello nos permitirá inferir en que medida las mujeres están igualitariamente representadas en los consejos. Estos datos pueden observarse en la columna derecha de la tabla 4. En relación al tamaño del propio consejo, puede observarse en términos generales que mientras mayor es el tamaño medio del consejo, mayor es el nivel de representación de la mujer. Este dato podría poner de manifiesto que aunque las mujeres ganan acceso al consejo de administración lo hacen a través del aumento del tamaño de los mismos, lo que explica la menor participación de éstas en términos relativos en la cúpula de poder organizativo.

\subsection{Análisis MULTIVARIANTE}

Al objeto de analizar como interactúan de forma conjunta todas las variables para explicar el nivel de presencia de las mujeres en los consejos de administración se ha estimado el modelo descrito en el apartado 3.1. Junto al valor que alcanzan los coeficientes de las variables y el nivel de significatividad de los mismos, se recogen determinados estadísticos que reflejan la bondad del ajuste alcanzado. En la tabla 5 se reflejan los resultados estimados para las distintas variables, experimentales y de control, cuando la variable dependiente es el porcentaje de mujeres representadas en el consejo de administración. En el análisis se ha controlado la adscripción sectorial de las empresas recogidas en la muestra. El método de estimación ha sido por mínimos cuadrados ordinarios y hemos tratado la posible multicolinealidad a la que se puede ver afectado nuestro modelo ${ }^{(12)}$.

TABLA 5

Resultados alcanzados para la variable PARTiCiPACión

\begin{tabular}{|l|c|c|c|c|}
\hline VARIABLES & MODELO 1 & MODELO 2 & MODELO 3 & MODELO 4 \\
\hline CONSTANTE & $7,75(0,000)$ & $3,936(0,000)$ & $10,47(0,000)$ & $0,81(0,417)$ \\
\hline CONSEJEROS & $1,85(0,064)$ & $1,90(0,058)$ & $4,69(0,000)$ & $3,21(0,000)$ \\
\hline ACTIVIDAD & $3,35(0,000)$ & $3,40(0,000)$ & $3,21,(0,000)$ & $2,34(0,000)$ \\
\hline RENTABILIDAD & $-0,33(0,739)$ & $-0,17(0,867)$ & $0,48(0,628)$ & $0,69(0,758)$ \\
\hline CODIGO & - & $4,65(0,000)$ & - & - \\
\hline BELGICA & - & - & $-8,03(0,000)$ & - \\
\hline DINAMARCA & - & - & $-6,63(0,000)$ & \\
\hline
\end{tabular}

(Continúa pág. sig.)

(12) Para evaluar el grado de multicolinealidad, hemos analizado el factor de inflación de la varianza (FIV) de todas las variables consideradas y en todos los casos se puede decir que no encontramos en nuestro modelo problemas relacionados con la multicolinealidad. La literatura suele recomendar valores inferiores a cinco para el FIV, mientras que en nuestro estudio todos los valores obtenidos son siempre inferiores a tres. 
TABLA 5 (CONT.)

Resultados alcanzados para la VARIable PARTICIPACIÓN

\begin{tabular}{|l|c|c|c|c|}
\hline ESPAÑA & - & - & $-7,86(0,000)$ & - \\
\hline INGLATERRA & - & - & $-9,27(0,000)$ & - \\
\hline DIPODER & - & - & - & $-5,39(0,000)$ \\
\hline INCERTIDUMBRE & - & - & - & $-4,87(0,000)$ \\
\hline INDIVIDUALISMO & - & - & - & $-0,28(0,781)$ \\
\hline MASCULINIDAD & - & - & - & $-5,01(0,000)$ \\
\hline Control sectorial & SI & SI & SI & SI \\
\hline R cuadrado & 0,018 & 0,039 & 0,140 & 0,140 \\
F-Test & 6,900 & 10,683 & 22,402 & 23,341 \\
$p$-value & 0,000 & 0,000 & 0,000 & 0,000 \\
\hline
\end{tabular}

En el análisis planteado se han estimado diversos modelos en los que se ha profundizado en las relaciones que son objeto de interés en este estudio. En el modelo uno se estima el modelo considerando únicamente las variables que en este estudio son consideradas como variables de control.

Como puede observarse en la tabla 5, dos variables adquieren capacidad explicativa, en concreto el tamaño del consejo y la variable actividad, de tal forma que el nivel de participación de mujeres en el consejo de administración es mayor para las empresas cuyos consejos de administración son mayores y para las empresas de mayor tamaño absoluto atendiendo al volumen de facturación. Estas evidencias ponen de manifiesto una serie de cuestiones importantes. En primer lugar, y en referencia al tamaño del consejo, puede afirmarse que la relación positiva que liga el tamaño del consejo y el porcentaje de representación pone de relieve que la mayor representación se obtiene a través de incrementar el número de miembros de los consejos de administración, es decir, los hombres no pierden representación en términos absolutos. Ello puede obedecer a que las empresas responden al debate sobre políticas de igualdad de género incrementando el número total de miembros del consejo, y no haciendo que éstos sean más paritarios. En segundo lugar, en relación a la variable que representa el tamaño de la empresa, la relación empírica que se detecta es positiva, lo que significa que las mujeres tienen más facilidad de acceso a los consejos de administración mientras mayor es el tamaño de la empresa. De esta forma, en aquellas empresas en las que existen procesos más formalizados de promoción, es decir las grandes empresas, las mujeres tienen menos dificultad de acceder a los puestos del consejo de administración. Estas relaciones empíricas se mantienen para los distintos modelos estimados, por lo que pueden ser consideradas robustas a distintas especificaciones del modelo.

En el modelo dos se introduce, sobre el modelo base, la variable CÓDIGO, al objeto de analizar si los esfuerzos institucionales realizados por los códigos de gobierno corporativo para incrementar la diversidad en los consejos de administración y, en especial, la mayor presencia de mujeres, tiene capacidad para explicar la mayor representación de mujeres en los consejos de administración. 
Los resultados que se obtienen revelan que el coeficiente de esta variable es positivo y significativamente distinto de cero, lo que en suma pone de manifiesto que los esfuerzos realizados en los países para crear una política de paridad en los consejos a través de una mayor incorporación de mujeres parecen resultar efectivos.

En el modelo tres se intenta analizar si la participación en el consejo de administración de las mujeres viene explicada por un efecto país; para ello se ha introducido diferentes variables dicotómicas para identificar el país de origen de las empresas (Belgica, Dinamarca, España e Inglaterra), actuando Suecia como categoría base frente a las que pretende analizarse el efecto país.

Como ya se avanzaba a nivel univariante las cuatro variables referidas a los países contemplados adquieren significado estadístico, siendo la relación que las une con la variable PARTICIPACIÓN negativa en todos los casos. Lo anterior pone de relieve que la procedencia del país está explicando el mayor o menor nivel de representación de mujeres en el consejo de administración de las empresas, y en todos los países el nivel de acceso de la mujer a los consejos de administración es menor que para el caso sueco.

Para profundizar sobre los aspectos singulares relacionados al país que pueden estar explicando estas diferencias en el nivel de presencia de las mujeres en los consejos de administración, se ha estimado el modelo cuatro en el cual se introducen las distintas dimensiones culturales que caracterizan a un país. Para ello utilizamos los valores que adquieren las diferentes dimensiones culturales de Hofstede (1980), lo que nos permitirá deducir si la cultura del país puede explicar el mayor progreso de las mujeres en los puestos de mayor responsabilidad de las empresas.

Como podemos apreciar, tres variables relacionadas a la cultura del país son significativas estadísticamente. La variable DIPODER es significativa y el signo del coeficiente es negativo, lo que revela que en aquellas sociedades más igualitarias y donde la distancia al poder es más baja hay una mayor proporción de mujeres representadas en el consejo de administración, siendo éste el resultado esperado. En lo que se refiere a la variable aversión de la incertidumbre (INCERTIDUMBRE), el coeficiente es significativamente distinto de cero y la relación que la une con la variable dependiente es negativa; este resultado confirma que las sociedades que aceptan mejor el cambio, los comportamientos alternativos y mayor propensión al riesgo facilitan más la representación de las mujeres en el consejo de administración. Por último, la variable MASCULINIDAD también adquiere significado estadístico y su relación con la variable dependiente es negativa, revelando que en aquellas sociedades donde prevalecen valores asociados al rol femenino existen un mayor nivel de representación de las mujeres en el consejo de administración.

Los resultados obtenidos nos permiten concluir como la cultura del país, calculada a través de las dimensiones culturales de Hofstede (1980), muestran capacidad para explicar las diferencias detectadas en el nivel de representación de mujeres en los consejos de administración, resultados éstos que permiten confirmar la importancia de la cultura como factor determinante del acceso de la mujer a los puestos de representación del consejo de administración. 


\section{ANÁLISIS DE ROBUSTEZ DE LOS RESULTADOS}

Al objeto de analizar la robustez de los resultados obtenidos en nuestro estudio se han planteado diversos análisis adicionales que nos permitan determinar que nuestros resultados no se ven afectados por la fórmula de cálculo de algunas de las variables utilizadas o por la composición de la muestra.

\subsection{Análisis de la robustez de los resultados Utilizando El Modelo GLOBE}

Como señala Robbins (2004) las dimensiones culturales de Hofstede se han convertido en el marco teórico básico para diferenciar culturas nacionales, a pesar de que los datos en que se basa proceden de una única compañía, en concreto IBM, y tienen alrededor de 30 años de antigüedad, lo cual puede reducir y erosionar la capacidad de explicar la diversidad cultural entre países. Ello resulta quizás más relevante si atendemos a los incesantes e intensos cambios geopolíticos que se han vivido en los últimos tiempos, entre los que resulta obligado destacar la caída de la Unión Soviética, la reunificación de Alemania, el final del apartheid en Sudáfrica y el ascenso de China como potencia mundial. Ello pone de manifiesto el carácter mutable de la cultura, por lo que la misma no puede ser considerada un constructo conceptual estructural y sin dinámica propia susceptible de cambiar a lo largo del tiempo (McSweeney, 2002; Shenkar, 2001; Smith et $a l ., 2002)$. Al objeto de verificar en que medida nuestros resultados pueden verse afectados por la métrica de cálculo de los aspectos determinantes de la cultura de un país, vamos a reproducir los resultados utilizando el modelo GLOBE.

En 2004 el programa de investigación GLOBE, en sus siglas originales Global Leadership Organizational Behavior Effectiveness (House et al., 2004) ${ }^{(13)}$, presentó los resultados de su investigación cuyo principal objetivo era describir, comprender y predecir la influencia de variables culturales sobre liderazgo, proceso de gestión y su efectividad en cualquier parte del mundo. Este programa comenzó en 1993, el cual utilizando datos de 825 organizaciones de 62 países identificó nueve dimensiones que fueron denominadas como: aversión a la incertidumbre; distancia en el poder; énfasis de la sociedad en el colectivismo; prácticas colectivista en familia y en grupo; igualdad de género; asertividad; orientación hacia el futuro; orientación a los resultados y orientación humana ${ }^{(14)}$. En

(13) Eficacia Mundial del Comportamiento Organizacional y el Liderazgo.

(14) Distancia de poder. Grado en que los miembros de una sociedad esperan que la distribución de poderes sea inequitativa (DISPODER).

Aversión a la incertidumbre. Dependencia de la sociedad de sus normas y procedimientos para atenuar la incertidumbre (INCERTID).

Orientación humana. Grado en que la sociedad fomenta y recompensa a los individuos para que sean justos, altruistas, generosos, interesados en los demás y amables (ORIENTACIÓN).

Colectivismo en grupos. Grado en que los miembros de una sociedad se enorgullecen de formar parte de grupos pequeños, como su familia y círculo íntimo de amistades, así como la organización para la que trabajan (COLGRUPAL).

Individualismo o colectivismo, también denominado colectivismo institucional. Grado en el que las instituciones sociales alientan a los individuos a integrarse en grupos dentro de las organizaciones y la sociedad (COLINSTITUCIONAL).

Asertividad. Grado en el que la sociedad alienta a las personas a ser duras, polémicas, afirmativas y competitivas a diferencia de ser modestas y suaves. (ASERTIVIDAD).

Orientación hacia el futuro. Grado en el que la sociedad estimula y premia las conductas orientadas al futuro, como planear, invertir en el futuro y demorar la gratificación (FUTURO).

Orientación al desempeño. Grado en que una sociedad estimula y premia a los miembros de los grupos por sus mejoras de desempeño y por la excelencia (RENDIMIENTO). 
una comparación de las dimensiones de GLOBE y las de Hofstede se muestran que aquéllas amplían el trabajo de éste, más que sustituirlo (House et al., 2004). El estudio de GLOBE confirma que las cinco dimensiones de Hofstede todavía son válidas; sin embargo, añade otras y aporta una medida actualizada de las calificaciones de varios países en cada dimensión. Además este estudio midió las nueve dimensiones usando escalas multiítems, que analizan la descripción de los encuestados sobre «cómo es» su cultura y «cómo debería ser». La escala de valoración es de 1 a 7 puntos, donde 7, indica mayor grado de la dimensión correspondiente. Los resultados obtenidos para la estimación por mínimos cuadrados ordinarios se recogen en la tabla 6.

TABLA 6

Resultados obTenidos UTILIZANDo las Dimensiones CUlturales de GLOBE PaRa EL PORCENTAJE DE MUJERES REPRESENTADAS EN EL CONSEJO DE ADMINISTRACIÓN

\begin{tabular}{|l|c|}
\hline VARIABLES & MODELO 5 \\
\hline CONSTANTE & $3,901(0,000)$ \\
\hline CONSEJEROS & $5,43(0,000)$ \\
\hline ACTIVIDAD & $1,69(0,090)$ \\
\hline RENTABILIDAD & $0,45(0,653)$ \\
\hline DISPODER & $-2,11(0,035)$ \\
\hline INCERTID & $-4,72(0,000)$ \\
\hline ORIENTACIÓN & $0,46(0,456)$ \\
\hline COLGRUPAL & $1,34(0,325)$ \\
\hline COLINSTITUCIONAL & $0,43(0,345)$ \\
\hline ASERTIVIDAD & $-4,56(0,000)$ \\
\hline FUTURO & $0,78(0,367)$ \\
\hline RENDIMIENTO & $0,56(0,789)$ \\
\hline IGUALGENERO & $3,22(0,000)$ \\
\hline Control sectorial & $\mathrm{SI}$ \\
\hline R cuadrado & 0,144 \\
F-Test & 25,091 \\
p-value & 0,000 \\
\hline
\end{tabular}

Solo se ha estimado un único modelo en el que se recoge las dimensiones culturales de GLOBE, el cual revela una serie de cuestiones importantes ${ }^{(15)}$.

En primer lugar hemos de señalar que como en el caso anterior existen determinadas variables que adquieren significado estadístico, en concreto distancia al poder, incertidumbre, asertividad e igualdad de género ${ }^{(16)}$, lo que confirma que las variables que determinan la cultura de un país, con independencia de la métrica que se utilice para

Igualdad de género. Grado en el que la sociedad atenúa las diferencias en los papeles atribuidos tradicionalmente a cada sexo (IGUALGENERO).

(15) Hemos de resaltar que para proceder a la realización de este análisis adicional hemos eliminado las observaciones referidas a Bélgica dado que no están disponibles los valores GLOBE para este país y hemos utililizado Los valores correspondientes a la descripción de cómo es su cultura, acorde con el trabajo de Hofstede.

(16) GLOBE no considera la dimensión masculinidad de Hofstede, sino que utiliza las dimensiones asertividad e igualdad de género en sustitución de aquella 
inferir ésta, explica el mayor nivel de representación de la mujer en los consejos de administración. En segundo lugar, los resultados parecen confirmar la correlación existente entre las dimensiones culturales de Hofstede (1980) y las de GLOBE (House et al., 2004). Todo ello nos permite inferir que nuestros resultados son robustos con independencia de la métrica utilizada para analizar los factores que determinan la cultura de un país.

\subsection{ANálisis DE LA ROBUSTEZ DE LOS RESUltados EN RELACión a LA MUESTRA}

El segundo análisis adicional pretende medir la robustez de nuestros resultados a la composición de la muestra, para lo cual vamos a reproducir el análisis primario (Modelo 4) para dos submuestras distintas. El primer análisis reduce el estudio únicamente en aquellas observaciones donde existe representación de mujeres en los consejos (Modelo 4.1. tabla 7). Con ello queremos analizar en qué medida nuestros resultados pueden verse afectados por aquellas observaciones en las que, con independencia de la cultura que prevalece en un país, las empresas muestran una fuerte resistencia a admitir el acceso de la mujer a los consejos de administración. El segundo análisis utiliza una submuestra donde se eliminan las observaciones referidas a Inglaterra (Modelo 4.2., tabla 7). Dado que en la elaboración de la muestra hemos utilizado un criterio de selección de las observaciones para el caso de Inglaterra más restrictivo, hemos procedido a analizar si nuestros resultados están afectados por este criterio de selección.

Los resultados que se obtienen una vez estimado el modelo en ambas submuestras se recogen en la tabla 7 .

\section{TABLA 7}

Análisis de robusteZ de los ReSUltados a la COMPOSICIÓn de LA MUESTRA

\begin{tabular}{|l|c|c|}
\hline VARIABLES & MODELO 4.1 & MODELO 4.2 \\
Coeficientes $(p$-value $)$ & Coeficientes $(p$-value) \\
\hline CONSTANTE & $3,13(0,002)$ & $0,23(0,814)$ \\
\hline CONSEJEROS & $6,53(0,000)$ & $2,24(0,025)$ \\
\hline ACTIVIDAD & $2,60(0,000)$ & $1,99(0,000)$ \\
\hline RENTABILIDAD & $0,95(0,341)$ & $0,61(0,542)$ \\
\hline DIPODER & $-4,27(0,000)$ & $-4,98(0,000)$ \\
\hline INCERTIDUMBRE & $-3,33(0,001)$ & $-3,72(0,000)$ \\
\hline INDIVIDUALISM0 & $-0,00(0,999)$ & $-1,23(0,219)$ \\
\hline MASCULINIDAD & $-2,13(0,033)$ & $-8,54(0,000)$ \\
\hline Control Sectorial & Sí & Sí \\
\hline R cuadrado & 0,171 & 0,117 \\
F-Test & 16,415 & 0,000 \\
\hline$p$-value & 0,000 & 17,615 \\
\hline
\end{tabular}

Los resultados obtenidos confirman los resultados primarios alcanzados, es decir, adquieren significado estadístico las mismas variables, lo que nos permite concluir que nuestros resultados son robustos y no están afectados por la forma en la cual se configura la muestra. 


\subsection{ANÁlISIS DE LA ROBUSTEZ DE LOS RESULTAdOS INTRODUCIENDO NUEVAS VARIABLES DEL ENTORNO INSTITUCIONAL DEL PAÍS}

La cultura es un factor institucional único dentro del contexto institucional de un país y diferenciable de otros elementos básicos del entorno como son los factores económicos, políticos, legales, educativos, etc. No obstante, resulta complejo determinar en que medida el fenómeno objeto de nuestro estudio viene únicamente explicado por la cultura de un país, dado que pueden existir otros factores institucionales que interrelacionados con la cultura pueden estar afectando al acceso de la mujer a los consejos de administración. Para verificar si los resultados obtenidos en nuestro estudio son robustos hemos introducido en el modelo otras variables definitorias del entorno institucional al objeto de analizar como afecta su interrelación con la cultura al acceso de la mujer a los consejos de administración. Para ello nos valemos del trabajo de Kauffman et al. (2009) ${ }^{(17)}$, el cual pretende medir las tradiciones e instituciones a través de la cual la autoridad es ejercida en un país. Para ello definen seis indicadores de gobierno. Responsabilidad (RESP) mide la extensión por la cual los ciudadanos de un país son capaces de participar en la selección de su gobierno. Estabilidad política (ESTAB) mide la probabilidad de que el gobierno sea estable. Efectividad del gobierno (EFECT) mide la calidad de los servicios públicos. Calidad de la regulación (CAL) mide la capacidad del gobierno para formular e implementar políticas adecuadas que posibiliten el desarrollo del país. Normas de ley (NORM) mide la confianza de los individuos en las normas de la sociedad. Control de la corrupción (CONT) mide si el poder público es ejercido para obtener ganancias privadas. Al margen de estas variables hemos introducido una variable del bienestar económico del país a través del producto interior bruto por individuos del país (PIB). Los resultados obtenidos se muestran en la tabla 8.

TABLA 8

ANÁlISIS DE ROBUSTEZ DE LOS RESULTADOS A LA COMPOSICIÓN DE LA MUESTRA

\begin{tabular}{|l|c|c|}
\hline VARIABLES & $\begin{array}{c}\text { MODELO 6 } \\
\text { Coeficientes }(p \text {-value })\end{array}$ & $\begin{array}{c}\text { MODELO 7 } \\
\text { Coeficientes }(p \text {-value })\end{array}$ \\
\hline CONSTANTE & $5,04(0,000)$ & $6,19(0,000)$ \\
\hline CONSEJEROS & $2,31(0,021)$ & $2,54(0,023)$ \\
\hline ACTIVIDAD & $2,18(0,029)$ & $2,98(0,015)$ \\
\hline RENTABILIDAD & $0,78(0,613)$ & $0,96(0,632)$ \\
\hline DIPODER & - & $-3,87(0,000)$ \\
\hline INCERTIDUMBRE & - & $-5,24(0,000)$ \\
\hline INDIVIDUALISMO & - & $-1,56(0,329)$ \\
\hline MASCULINIDAD & - & $-2,74(0,000)$ \\
\hline RESP & $1,04(0,654)$ & $0,67(0,856)$ \\
\hline ESTAB & $5,36(0,000)$ & $5,39(0,000)$ \\
\hline EFECT & $0,87(0,876)$ & $1,21(0,134)$ \\
\hline CAL & $1,34(0,129)$ & $2,17(0,054)$ \\
\hline
\end{tabular}

(Continúa pág. sig.)

(17) Agradecemos la sugerencia que sobre este extremo nos ha realizado uno de los evaluadores del trabajo. 
TABLA 8 (CONT.)

ANÁlisis de robustez de los Resultados a la COMPOSICIÓn de LA MUESTRA

\begin{tabular}{|l|c|c|}
\hline NORM & $1,19(0,234)$ & $2,13(0,000)$ \\
\hline CONT & $2,74(0,006)$ & $3,21(0,000)$ \\
\hline PIB & $-4,55(0,000)$ & $-2,75(0,000)$ \\
\hline Control sectorial & $\mathrm{SI}$ & $\mathrm{SI}$ \\
\hline$R$ cuadrado & 0,129 & 0,182 \\
F-Test & 21,075 & 23,235 \\
$p$-value & 0,000 & 0,000 \\
\hline
\end{tabular}

Como puede observarse en dicha tabla hemos regresado dos nuevos modelos. El primer modelo introduce únicamente estas nuevas variables institucionales como determinantes del acceso de la mujer a los consejos de administración. En el segundo modelo hacemos interactuar estas nuevas variables con las dimensiones culturales de Hofstede.

De los resultados recogidos en la tabla 7 se puede deducir que otras variables del entorno institucional del país tienen capacidad para explicar el diferente nivel de acceso de la mujer a los consejos de administración cuando se comparan países distintos, si bien, al objeto de este estudio lo que resulta importante destacar es que los resultados obtenidos respecto a las dimensiones culturales no se ven afectados. En este sentido, los indicadores de la cultura de un país son diferenciables del resto de indicadores del entorno institucional y tienen capacidad para explicar el diferente acceso de la mujer a los consejos de administración de los distintos países objeto de nuestro trabajo.

\section{CONCLUSIONES}

La tesis que se sustenta en este trabajo es que el diferente nivel de representación de mujeres en los consejos de administración de las empresas cuando se comparan países distintos viene explicado en gran parte por el papel de la cultura que prevalece en cada país, dado que ésta puede crear estereotipos de roles asociados a hombres y mujeres que pueden afectar al acceso de la mujer a los consejos de administración.

Como ponen de manifiesto numerosos estudios existen diferencias importantes en la cultura existente entre países (Hofstede, 1980; Schwartz, 1992; Ingleharts, 1977, 2001; Trompenaars, 1993; House et al., 2004), y como apoya la teoría institucional, estas diferencias culturales son la base para las divergencias en las estructuras de gobierno de las empresas a nivel internacional.

Para contrastar nuestra tesis, estimamos un modelo en el que las variables experimentales son las cuatro dimensiones culturales identificadas por Hofstede (1980), y desarrollamos un análisis estadístico descriptivo, univariante y multivariante sobre una muestra de empresas de cinco países. Los resultados obtenidos poden de relieve como la cultura que prevalece en estos países tiene capacidad para explicar el diferente nivel de representación de las mujeres en los consejos de administración. Tres de las cuatro dimensiones culturales de Hofstede se revelan como significativas: 
distancia en el poder, de tal forma que aquellas sociedades más igualitarias y donde la distancia al poder es más baja hay una mayor proporción de mujeres representadas en el consejo de administración; aversión a la incertidumbre, concluyéndose que aquellas sociedades que aceptan mejor el cambio, los comportamientos alternativos y mayor propensión al riesgo, facilitan más la representación de las mujeres en el consejo; y por último, masculinidad, poniéndose de manifiesto que en aquellas sociedades donde prevalecen valores asociados al rol femenino existen un nivel de representación de las mujeres en los consejos de administración mayor.

Estos resultados se ven confirmados con los obtenidos introduciendo las nueve dimensiones culturales de GLOBE (House et al., 2004), y otros indicadores del entorno institucional lo que nos lleva a concluir que las variables que determinan la cultura de un país, con independencia de la métrica que se utilice, son diferenciables del resto de indicadores del entorno institucional y tienen capacidad para explicar el mayor o menor nivel de representación de la mujer en los consejos de administración y por tanto, es un factor determinante del acceso de la mujer a los puestos de responsabilidad de las empresas.

Por último, una importante implicación que surge de nuestro estudio es que dado que la cultura, como sistema de creencias y valores sociales tienden a ser inamovibles a corto plazo, si desde el punto de vista social se considera adecuado que hombres y mujeres estén representados de forma similar en los consejos de administración de las empresas, se requerirán de esfuerzos institucionales importantes para conseguir cambios en los niveles de acceso de la mujer en los puestos de responsabilidad de las empresas, cuestión ésta que parece quedar constatada en los momentos actuales por las políticas de paridad por género y los esfuerzos que los diversos gobiernos están llevando a cabo en este sentido.

Como limitación a nuestro estudio señalar que hemos realizado una comparación sólo de cinco países, por lo que nuestros resultados deben ser considerados en relación a los mismos, y por tanto no pueden ser extrapolados a otros países. A la extensión del número de países a someter a estudio dedicaremos futuros trabajos.

\section{BIBLIOGRAFÍA}

AdLER, R. D. 2001. Women in the executive suite correlate to high profits. Working paper. European Project on Equal Pay.

Aguilera, R. V., y Jackson, G. 2003. The cross-national diversity of corporate governance dimensions and determinants. Academy of Management Journal, 28(3): 447-465.

ANDRE, R. 1995. Diversity in executive networks: a national study of women's representation in private sector economic development. Journal of Managerial Issues 7 (3): 306-322.

BAysinger, B. D., y Butler, H. N. 1985. Corporate governance and the board of directors: Performance effects of changes in board composition. Journal of Law, Economics and Organization, 1: $101-124$.

Bertrand, M., y Hallock, K. F. 2001. The gender gap in top corporate jobs. Industrial \& Labor Relation Review, 55 (1): 3-21. 
Bhagat, S., y Black, B. 2000. Board Independence and long-term firm performance. Working paper. University of Colorado.

BREWER, B. 2001. Women building presence in non-traditional fields. Business Woman, Spring: 11-40.

BuRke, R. J. 1997. Women on Corporate Boards of Directors. A needed resource, Journal of Business Ethics, 16 (9): 909-915.

Carrasco, A., y Laffarga, J. 2008. Diversidad en Gobiernos Corporativos y Flexibilidad en Sistemas Contables de los Paises Europeos. Working paper. VI Worshop de Investigación Empírica en Contabilidad Financiera y III Jornada de Investigación de la REFC. Madrid. Marzo 2008.

Carter, D. A.; Simkins, B. J., and Simpson, W. G. 2003. Corporate Governance, Board Diversity, and Firm Value. The Financial Review 38: 33-53.

Catalyst. 2001. 2001 Catalyst Census of Women Board Directors. New York, NY: Catalyst, .

- 2004. The Bottom Line: Connecting Corporate Performance and Gender Diversity. New York. www.catalystwomen.org

- 2008. Catalyst Census of women Board Directors of the Fortune 500. New York: www.catalystwomen.org

CNMV. Comisión Nacional del Mercado de Valores. 2006. Informe del grupo especial de trabajo sobre buen gobierno de las sociedades cotizadas. http://www.cnmv.es/index.htm

Coffey, B. S., y WANG, J. 1998. Board Diversity and Managerial Control as Predictors of Corporate Social Performance. Journal of Business Ethics 17: 1.595-1.603.

DAily, C. M., y DALTON, D. R. 1995. CEO and director turnover in failing firms: an illusion of change? Strategic Management Journal, 16: 393-400.

DiMaggio, P. J., y Powell, W. (1983). The iron carge revisited insituttional isomorphism and collective rationality in organizational fields. American Sociological Review 48: 147-160.

Erez, M., y EARLY, P. 1993. Culture, self-identity and work, Oxford: Oxford University Press.

European Proffesional Women's Network (EPWN). 2004. The First EPWN European Board Women Monitor. 2004. www.Europeampwn.net-

- 2008. 3. ${ }^{\text {rd }}$ EuropeanPWN Board Women Monitor 2008. www.Europeanpwn.net

Erhardt, N. L.; Werbel, J. D., y Shrader, C. B. 2003. Board of Director Diversity and Firm Financial Performance. Corporate Governance 11 (2): 102-111.

FARRell, K. A., y Hersch, P. L. 2005. Additions to corporate boards: The effect of gender. Journal of Corporate Finance 11: 85-106.

Friedland, R., y Alford, R. R. 1990. Bringing society back in Symbols, practices and institutional contradictions. In W. W. Powell and P. J. Di Maggio (eds.). The New Institutionalism in Organization Analysis, 232-263. Chicago, IL: University of Chicago Press.

Gray, S. J. 1988. Towards a Theory of Cultural Influence on the Development of Accounting Systems Internationally. ABACUS March: 1-15.

Guiso, L.; Sapienza, P., y Zingales. L. 2006. Does Culture Affect Economic Outcomes? Social Science Research Network. CEPR Discussion Paper n. ${ }^{\circ} 5.505$.

Hambrick, D. C., y CAnnella, A. A. 2004. CEOs who have COOs: Contingency analysis of an unexplored structural form. Strategic Management Journal 25: 959.

Harrigan, K. R. 1981. Numbers and positions of women elected to corporate boards. Academy of Management Journal 24 (3): 619-625. 
Heidrick y Struggles. 1977. The changing Board Profile of the Board of Directors. Chicago, IL: Heidrick and Struggles.

- 2005. Corporate Governance in Europe: what's the outlook? 2005 study. Heidrick Struggles International, Inc.

- 2007. Corporate Governance in Europe: raising the bar. 2007 report. Heidrick Struggles International, Inc.

Hermalin, B., y Weisbach, M. S. 1988. The determinants of board composition. Rand Journal of Economics 19 (4): 589-606.

- 1991. The effects of Board Composition and Direct incentives on Firm Performance. Financial Management 20 (4): 101-112.

Hickson, D. J., y Pugh, D. S.1995. Management Worldwide: The Impact of Societal Culture on Organizations Around the Globe. London: Penguin.

Hillman, A. J., y Cannella, A. 2007. Organizational Predictors of women on Corporate Boards. Academy of Management Journal 50 (4): 941-952.

Hillman, A. J.; Cannella, A. A., y Harris, I. C. 2002. Women and minorities in the boardroom: How do directors differ? Journal of Management 28: 747-763.

HoFstede, G. 1980. Culture's consequences: International differences in work-related values. Beverly Hills, C.A: Sage.

- 1991. Cultures and organizations; Software of the mind. London: Mc-Graw-Hill.

- 2000. Cultures Consequences: Comparing Values, Behaviours, Institutions, and Organizations Across Nations. London: Sage Publications.

Hope, O-K. 2003. Disclosure Practices, Enforcement of Accounting Standards, An Analysts' Forecast Accuracy: An International Study. Journal of Accounting Research 41: 235-272.

House, R. J.; Hanges, P. J.; Ruiz-Quintanilla, S. A.; Dorfman, P. W.; Javidan, M., y Dickson, M. W. 1999. Cultural influences on leadership and organizations: Project GLOBE. In W. H. Mobley, M. J. Gessner y V. Arnold (eds.), Advances in global leadership. Greenwich, CT: JAI, 171-233.

House, R. J.; Hanges, P. J.; Javidan, M.; Dorfman, P. W., y Gupta, V. 2004. Culture, Leadership, and Organizations. The Globe Study of 62 Societies. London: Sage Publications.

INGLEHARTS, R. 1977. The silent revolution changing values and political styles among western publics. Pricenton, N.J.: Princeton University Press.

- 2001. Modernización y posmodernización: El cambio cultural, económico y político en 43 sociedades. CIS. 2001.

- 2004. Human Beliefs and Values. A cross-cultural sourcebook based on the 1999-2002 values surveys. México: Siglo XXI Editores.

Kaufmann, D.; Kraay, A., y Mastruzzy, M. 2009. Governance Matters VIII. Agregate and Individual Governance Indicators 1996-2008. Policy Research Working Paper 4978. The World Bank Development Research Group. Macroeconomics and Growth Team. June 2009.

Konrad, A. M., y MANGEL, R. 2000. The impact of work-life programs on firm productivity. Strategic Management Journal 21: 1.225.

Konrad, A. M.; Ritchie, J. E.; Lieb, P., y Corrigal, E. 2000. Sex differences and similarities in job attribute preferences: A meta-analysis. Psychological Bulletin 126: 593-641.

Ley Orgánica de Igualdad. 2007. Ley Orgánica 3/2007, de 22 de marzo, para la igualdad efectiva de mujeres y hombres. BOE n. ${ }^{\circ} 71$ de 23 de marzo de 2007. 
LI, J., y HARRISON, J. R. 2008. National Culture and the Composition and Leadership Structure of Boards of Directors. Corporate Governance, 16 (5): 375-385.

Litz, R. A., y Folker, C. A, 2002. When he and she sell seashells: Exploring the relationship between management team gender-balance and small firm performance. Journal of Developmental Entrepreneurship 7 (4): 341-359.

Lubatkin, M. H.; Lane, P. J.; Collin, S. O., y Very, P. 2005. Origins of corporate Governance in the USA, Sweden and France. Organization Studies 26 (6): 867-888.

- 2007. An embeddedness framing of governance and opportunism: towards a cross-nationally accommodating theory of agency. Journal of Organizational Behaviour 28: 43-58.

McCormick Hyland, M. A., y Marcellino, P. A. 2002. Examining gender on corporate boards: a regional study. Corporate Governance 2: 24-31.

McDonal, M. 2000. A start-up of her own. US News and World Report 128 (19): 34-42.

McSweEney, B. 2002. Hofstede's model of national cultural differences and their consequences: A triumph of faith \& a failure of analysis. Human Relations, 55 (1): 89-118.

Meyer, J. W., y Rowan, B. 1977: Institutionalized organizations; formal structures as myth and ceremony. American Journal of Sociology 83: 364-385.

Nelson, T., y Levesque, L. L. 2007. The status of women in corporate governance in high-growth, high-potential firms. Entrepreneurship Theory and Practice. March: 209-232.

OAKLEY, J. 2000. Gender-based barriers to senior management positions: understanding the scarcity of female CEOs. Journal of Business Ethics 27 (4): 321-334.

Peng, M. W. 2004. Outside directors and firm performance during institutional transitions. Strategic Management Journal 25: 453-471.

Ralston, D. A.; Gustafsoconsejo n, D. J.; Cheung, F. M., y Terpstra, R. H. 1993. Differences in managerial values: A study of US, Hong Kong and PRC managers. Journal of International Business Studies 24: 249-275.

Roвbins, S. 2004. Comportamiento Organizacional. México: Pearson Educación.

Rosenstein, S., y Wyatt, J. G. 1990. Outside directors, board independence and shareholder wealth. Journal of Financial Economic, 26, 175-191.

- 1997. Inside directors, board effectiveness, and shareholder wealth, Journal of Financial Economics 44: 229-250.

SAlter, S. B., y Niswander, F. 1995. Cultural influence on the development of accounting systems internationally: a test of Gray's, 1988 theory. Journal of International Business Studies 26: 379-397.

SANDER, W., y BoIvie, S. 2004. Sorting things out: Valuation of new firms in uncertain markets. Strategic Management Journal, 25, 167-186.

Schuler, R. S., y Rogovsky, N. 1998. Understanding compensation practice variation across firms: The impact of national culture. Journal of International Business Studies 29: 159-177.

Scoтt, W. R. 1987. Organizations: Rational, natural and open systems. Englewood Cliffs, NJ: Prentice Hall.

Schwartz. S. H. 1992. Universals in the content and structure of values: Theoretical advances and emprirical tests in 20 countries. Advances in Experimental social Psycholog 25: 1-65.

Schwartz, S. H. 1994. Beyond individualism/collectivism: new cultural diemnsions of values. In Kim, U.; Triandis, H. C.; Kagitcibasi, C.; Choi, S., y Yoon, G. (eds.), Individualism and Collectivism. Thousand Oaks, CA: Sage. 
SCHWARTZ y HARRISON. 1986. Beyond the first generation of women directors: on the other side of the roadblock. Directors and Boards 11 (1): 39-41.

Semenov, R. 2000. Cross-country differences in economic governance: culture as a major explanatory factor. Unpublished Doctoral Dissertation. Tilburg University. En HoFsTEDE, G., 2000. Cultures Consequences: Comparing Values, Behaviours, Institutions, and Organizations Across Nations. London: Sage Publications.

Shenkar, O. 2001. Cultural distance revisited: Towards a more rigorous conceptualization and measurement of cultural differences. Journal of International Business Studies 32 (3): 519-536.

Singh, V.; Kumra, S., y Vinnicombe, S. 2002. Gender and impression management: playing the promotion game. Journal of Business Ethics 37 (1): 77-89.

Sмiтн, E. 2007. Gender influence on firm-level entrepreneurship through the power structure of boards. Women in Management Review 22 (3): 168-186.

Smith, P. B.; Peterson, M., y Schwartz, S. 2002. Cultural values, sources of guidance, and their Relevance to Managerial Behavior. Journal of Cross-cultural Psychology, 33 (2): 188-208.

Stulz, R. M., y Williamson, R. 2003. Culture, openness and Finance. Journal of Financial Economics, 70 (3): 313-349.

Terjesen, S.; Sealy, R., y Singh, V. 2009. Women Directors on Corporate Boards: A Review and Research Agenda. Corporate Governance: An International Review 17 (3): 320-337.

TrompenaArs, F. 1993. Riding the Waves of Culture: Understanding Cultural Diversity in Business. London: Nicholas Brealey.

Very, P.; Lubatkin, M.; Calori, R., and Veiga, J. 1997. Relative standing and the performance of recently acquired European firms. Strategic Management Journal 18 (8): 593-614.

WeISBACH, M. S. 1988. Outside directors and CEO turnover. Journal of Financial Economics 20: 431-460.

Zelechoski, D., y Bilimoria, D. 2004. Characteristics of women and men corporate inside directors in the US. Corporate Governance: An International Review 12 (3): 337-342. 